\title{
AC CONDUCTIVITY OF COBALT PHTHALOCYANINE
}

\author{
T.G. AbDel-MaliK ${ }^{a}$, M.E. KASSEM ${ }^{b}$, N.S. Aly $^{c}$ AND S.M. KhaliL ${ }^{b}$ \\ ${ }^{a}$ Physics Department, Faculty of Science, El-Minia University, Egypt \\ ${ }^{b}$ Department of Physics, Faculty of Science, Alexandria University, Egypt \\ ${ }^{c}$ Department of Physics, Faculty of Education, Alexandria University, Egypt
}

(Received January 6, 1992; revised version April 3, 1992)

The dielectric properties of cobalt phthalocyanine are investigated in the frequency range $50-5 \times 10^{5} \mathrm{~Hz}$ and within the temperature range 293-363 K. The frequency dependence of the $\mathrm{AC}$ impedance could be expressed by the complex plane of the complex impedance. Analysis of the AC conductivity reveals semiconducting features based predominantly on the hopping mechanism. The relaxation time, derived from the experimental results, shows a thermally activated process.

PACS numbers: $77.40 .+\mathrm{i}$

\section{Introduction}

Organic semiconductors, like phthalocyanine, have attracted interest due to the low-dimensional nature of their electrical conduction and the current interest in solar energy conversion [1]. Cobalt phthalocyanine, being thermally and chemically stable, has been used as a material interesting from biological and chemical points of view. In this paper, we report on the dielectric properties of cobalt phthalocyanine at different temperatures.

\section{Experiment}

Cobalt phthalocyanine ( $\mathrm{CoPc}$ ) was purchased from Estman Kodak Ltd., New York. The starting material was carefully purified by vacuum sublimation in a two-zone tube furnace using the method previously described [2]. The purified material was pressed under the pressure of $9.8 \times 10^{8} \mathrm{~Pa}$ to form discs of diameter of $10 \mathrm{~mm}$ and thickness of 1 to $2 \mathrm{~mm}$. Contacts were made using silver electrodes. The sample was placed in a holder designed to minimize stray capacitance. The temperature dependence of the dielectric properties was measured using Tesla BM 507 impedance meter. The measurements were performed in the frequency 
range $50-5 \times 10^{5} \mathrm{~Hz}$ and temperature range 293-363 $\mathrm{K}$. The temperature was measured using a copper-constantan thermocouple mounted in close proximity to the specimen of interest.

\section{Results and discussion}

Many dielectric functions have been used to describe the frequency-dependent properties of material. Among them the most important ones are the complex dielectric constant $\left(\varepsilon^{*}\right)$, complex impedance $\left(Z^{*}\right)$ and complex conductivity $\left(\sigma^{*}\right)$. These functions may be expressed in terms of $\varepsilon^{*}$ as

$$
\begin{aligned}
\varepsilon^{*} & =\varepsilon^{\prime}-\mathrm{i} \varepsilon^{\prime \prime}, \\
Z^{*} & =Z^{\prime}-\mathrm{i} Z^{\prime \prime}=\frac{1}{\mathrm{i} \omega C_{0} \varepsilon^{*}}, \\
\sigma^{*} & =\frac{\varepsilon_{0}}{Z^{*} C_{0}},
\end{aligned}
$$

where $\mathrm{i}=\sqrt{-1}, \omega$ is the angular frequency, $2 \pi \nu$, and $C_{0}$ is the geometrical capacitance which is given for a disc by the vacuum permittivity $\varepsilon_{0}$, area $A$ and thickness $t$ in the relation

$$
C_{0}=\frac{\varepsilon_{0} A}{t}
$$

The measured impedances $Z(\nu)=Z^{\prime}(\nu)+\mathrm{i} Z^{\prime \prime}(\nu)$ were plotted in the complex plane for various temperatures. Typical spectra related to the frequency of the applied sinusoidal voltage are shown in Fig. 1. The plots become progressively
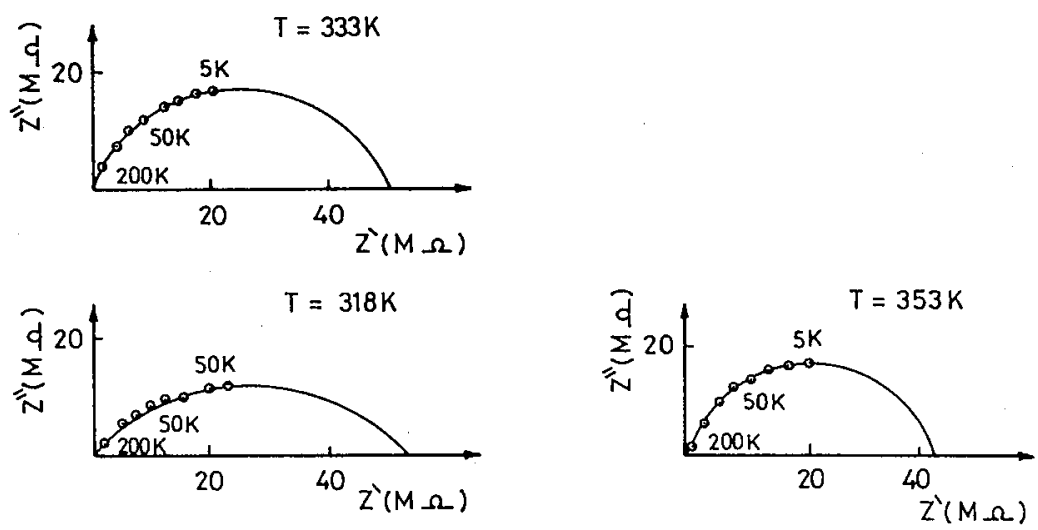

Fig. 1. Complex impedance spectra of CoPc for a range of temperatures. The frequencies indicated are in hertz.

more circular as the temperature increases. The classical semicircular form can be represented by a simple parallel $R_{\mathrm{s}}-C_{\mathrm{s}}$ circuit [3]. The capacitance

$$
C_{\mathrm{s}}=\lim _{\nu \rightarrow \infty}\left[-2 \pi \nu Z^{\prime \prime}(\nu)\right]^{-1}
$$


is the capacitance of the measured sample whereas the resistance

$$
R_{\mathrm{s}}=\lim _{\nu \rightarrow 0} Z^{\prime}(\nu)
$$

is the grain surface resistance.

The grain surface resistance $R_{\mathrm{s}}$ could be figured out from the intersection of the low-frequency semicircles with the $Z^{\prime}$-axis. The calculated values could be employed to shed light on the variation of the DC conductivity $\sigma_{\mathrm{DC}}$ of CoPc with temperature. Figure 2 shows a linear plot of $\log \sigma_{\mathrm{DC}}$ versus $1 / T$. An activation

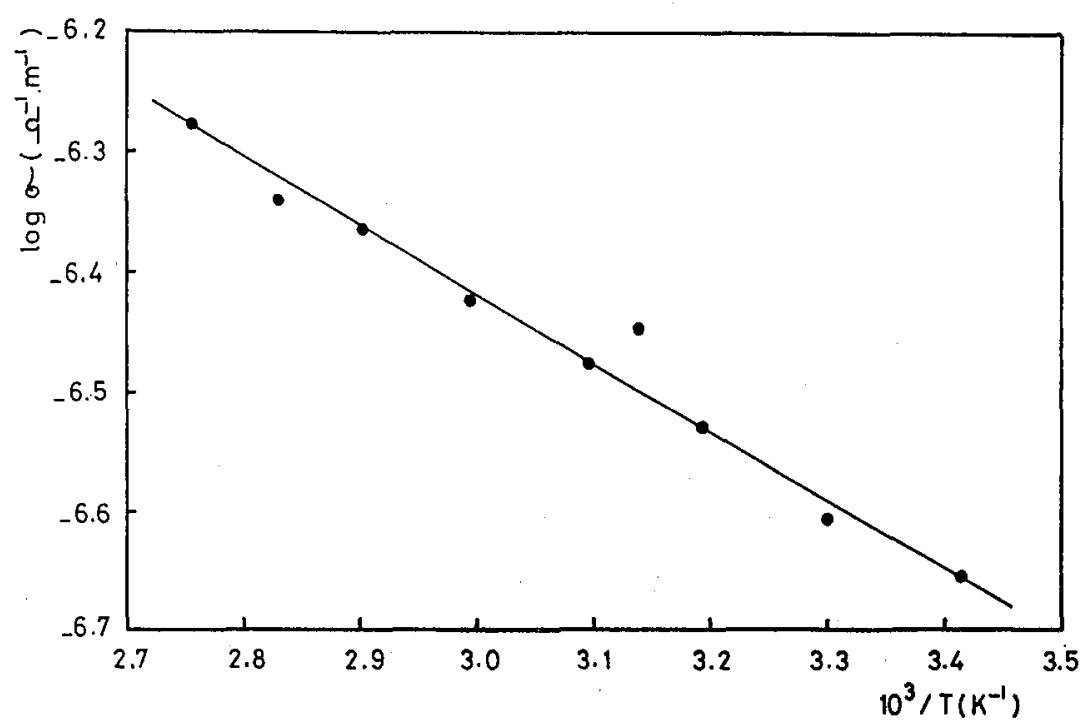

Fig. 2. Temperature dependence of DC conductivity of CoPc.

energy $E=0.11 \pm 0.02 \mathrm{eV}$ was obtained from the slope of the straight line using the well-known relationship $\sigma_{\mathrm{DC}}=\sigma \exp (-E / k T)$. The slope and the standard deviation were computed using a least-squares fit programme.

The frequency dependence of the conductivity for a range of temperatures is shown in Fig. 3. The conductivity obeyed the empirical law of frequency dependence given by the power law of the form [4]:

$$
\sigma(\omega)=\sigma_{\mathrm{DC}}+\text { const } \cdot \omega^{n} \text {. }
$$

The variation of the exponent $n$ with temperature gives information on the specific mechanism involved. As it can be seen from Fig. $3, n$ changes slightly with temperature, its value was found to be in the region $0.7-0.8$ suggesting a hopping mechanism of conduction [5]. This view is found in high-purity CoPc [6] where the $\mathrm{AC}$ conductivity was measured and the hopping mechanism of the charge carrier transport was uncritically concluded, while there is no other evidence to support such a statement and often there is ample evidence that this cannot be the case.

Further analysis of the $\mathrm{AC}$ conductivity data shows a simple activation energy, i.e. a straight-line segment on the plot of $\log \sigma_{D C}$ versus $1 / T$. The value of 


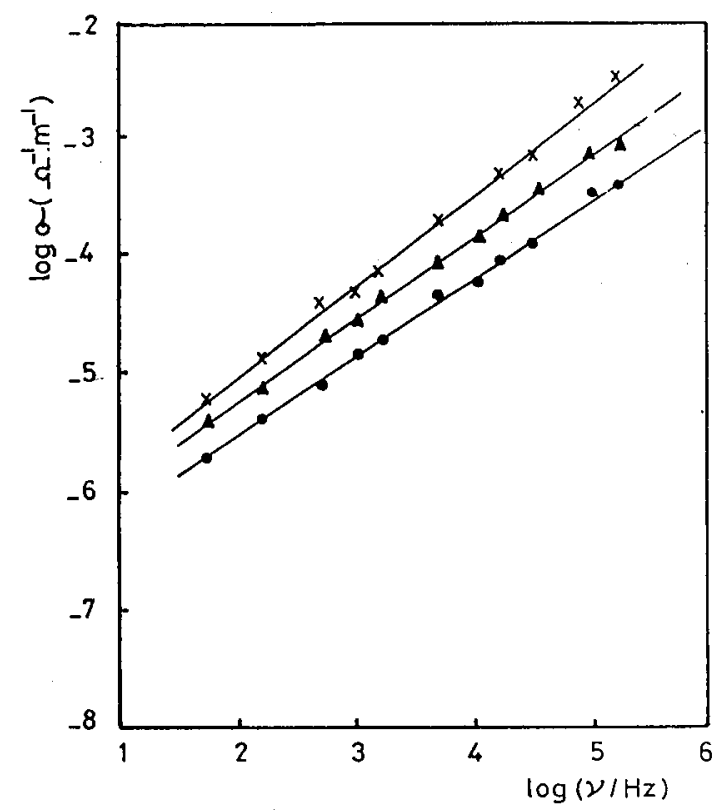

Fig. 3. The frequency dependence of the conductivity of CoPc for a range of temperatures. $T=\bullet 293$, full triangles $323, \times 353 \mathrm{~K}$.

the activation energy for $\sigma_{\mathrm{DC}}$ was found to be $0.08 \pm 0.01 \mathrm{eV}$. This value is not in agreement with the value reported by Wacławek et al. [6] for CoPc. However, a great number of localized states is expected to exist in phthalocyanine, but their density is incapable of dominating the statistics.

The dependence of the dielectric constant $\varepsilon^{\prime \prime}\left(\varepsilon^{\prime}\right)$ at different temperatures is shown in Fig. 4. A semicircle is obtained for each temperature. The centres of the semicircles lie below the abscissa axes. This confirms that there exists a distribution of relaxation times in CoPc. The analysis of the results shown in Fig. 4 could reveal several interesting parameters such as distribution parameter $\alpha$, relaxation time $\tau$ and activation energy $E_{0}[7,8]$. The macroscopic relaxation time $\tau_{0}$ can be evaluated from the relation

$$
\frac{U}{V}=\left(\omega \tau_{0}\right)^{1-\alpha} .
$$

Some of these symbols are shown in Fig. 4.

The parameter $\alpha$ is equal to zero when the dielectric has only one relaxation time. Whereas, for a distribution of relaxation times it varies between 0 and 1 . The extent of the distribution of relaxation times increases with increasing parameter $\alpha$.

The value of $\tau_{0}$ was found to decrease with increasing temperature. The molecular relaxation time $\tau$ could be evaluated from [8]:

$$
\tau=\frac{2 \varepsilon_{0}+\varepsilon_{\infty}}{3 \varepsilon_{0}} \tau_{0}
$$

where $\varepsilon_{0}$ is the static dielectric constant and $\varepsilon_{\infty}$ is the optical dielectric constant. 

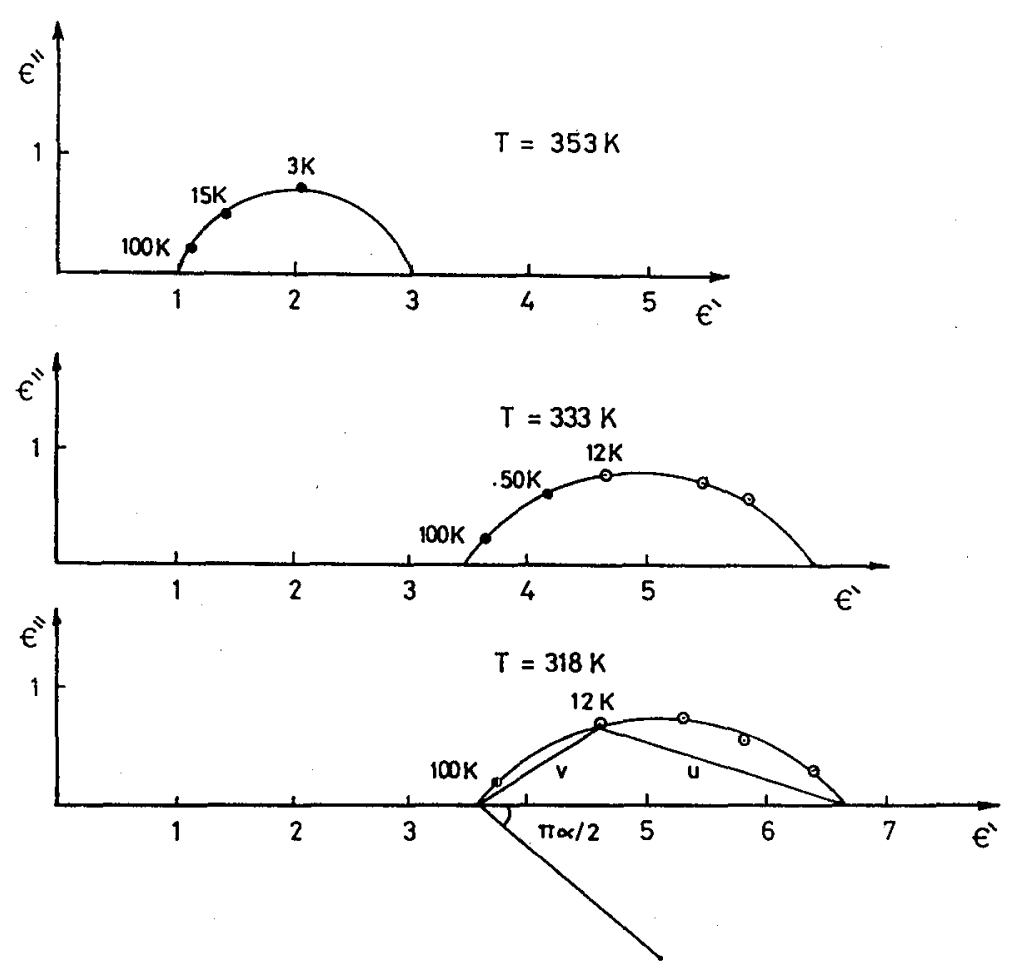

Fig. 4. Complex permittivity diagrams for a range of temperatures. The frequencies indicated are in hertz.

The temperature dependence of $\tau$ can be expressed by the thermally activated process of the type [9]:

$$
\tau=A \exp \left(E_{0} / k T\right)
$$

where $A$ is a constant characteristic relaxation time and represents the time of a single oscillation of a dipole in the potential well. $E_{0}$ is the free energy of activation for dipole relaxation. $\tau$ represents the average or most probable value of a spread of relaxation time.

Figure 5 shows the plot of $\log \tau$ as a function of $1 / T$. The values of $A$ and $E_{0}$ were found to be $A=(1.68 \pm 0.01) \times 10^{-6} \mathrm{~s}$ and $E_{0}=0.06 \pm 0.01 \mathrm{eV}$, respectively.

Both the molecular relaxation time $\tau$ and conductivity $\sigma(\omega)$ have the same activation energy for a thermally activated process since they depend on the temperature much more strongly than the other parameters, i.e. $\varepsilon_{0}, \varepsilon_{\infty}$, and $\alpha$ [10].

In the light of previously published results [11] inhomogeneities existing at grain boundaries in some organic materials restrict the movement of hopping carriers resulting in a decrease of dielectric losses. We may assume that the restriction of the carrier movement may be supposed to play a significant role in $\mathrm{CoPc}$ pellets. 


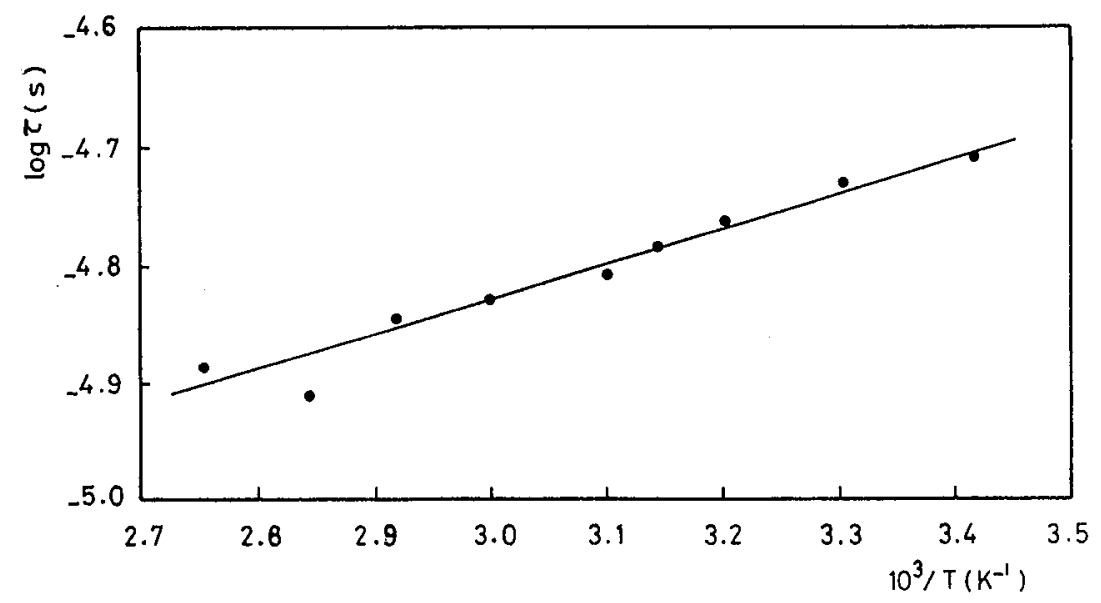

Fig. 5. Temperature dependence of molecular relaxation time of CoPc.

The following conclusions may be drawn from the afore-mentioned results:

(1) The AC conductivity in $\mathrm{CoPc}$ reveals semiconducting features based predominantly on the hopping mechanism.

(2) The temperature dependence of molecular relaxation time shows a thermally activated process.

\section{References}

[1] T.G. Abdel-Malik, A.A. Ahmed, A.S. Riad, Phys. Status Solidi A 121, 507 (1990).

[2] T.G. Abdel-Malik, G.A. Cox, J. Phys. C 10, 63 (1977).

[3] J.L. Carpentier, A. Lebrum, F. Perdu, J. Phys. Chem. Solids 90, 145 (1989).

[4] A.K. Jonscher, Dielectric Relaxation in Solids, Chelsea Dielectrics Press Ltd., London 1983.

[5] S.R. Elliott, Solid State Ion. Diffus. React. 27, 131 (1988).

[6] W. Wacławek, M. Kulesza, M. Ząbkowska, Mater. Sci. (Poland) VII, No 2-3, 385 (1981).

[7] R.H. Cole, K.S. Cole, J. Chem. Phys. 9, 314 (1941).

[8] K.K. Srivastava, A. Kumaro, O.S. Panwar, K.MN. Lakshminarayan, J. Non-Crystal. Solids 33, 205 (1979).

[9] R. Kawashima, M. Satoh, J. Phys. Soc. Jpn. 59, 3635 (1990).

[10] W. Cao, R. Gerhart, Solid State Ion. Diffus. React. 42, 213 (1990).

[11] G.W. Bak, A. Szymanski, A.K. Jonscher, J. Chem. Soc. Faraday Trans. II 81, 1053 (1985). 